\title{
Automated substance testing for lab-on-chip devices
}

\author{
Lutz Kloke ${ }^{1 *}$, Katharina Schimek' ${ }^{1}$ Sven Brincker ${ }^{1}$, Alexandra Lorenz ${ }^{1}$, Annika Jänicke ${ }^{1}$, Christopher Drewell ${ }^{1}$, \\ Silke Hoffmann ${ }^{1}$, Mathias Busek², Frank Sonntag ${ }^{2}$, Norbert Danz ${ }^{2}$, Christoph Polk², Florian Schmieder ${ }^{2}$, \\ Alexey Borchanikov ${ }^{4}$, Viacheslav Artyushenko ${ }^{4}$, Frank Baudisch ${ }^{3}$, Mario Bürger ${ }^{3}$, Reyk Horland ${ }^{1}$, Roland Lauster ${ }^{1}$, \\ Uwe Marx ${ }^{1}$
}

From 23rd European Society for Animal Cell Technology (ESACT) Meeting: Better Cells for Better Health Lille, France. 23-26 June 2013

\section{Background}

A smartphone-sized multi-organ-chip has been developed by TissUse. This platform consists of a microcirculation system which contains several fully endothelial-cellcoated micro- channels in which organ equivalents are embedded. Briefly, Human 3D organ equivalents such as liver and skin could be maintained functional over 28 days and treated with chemical entities in this microcirculation system.

In order to automate the Multi-Organ-Chip (MOC) handling we developed with partners a robotic platform. The prototype is capable to maintain 10 MOCs. Operations can be programmed individually by its user. For example OECD guidelines for acute toxicity testing could be performed. The robotic platform features also functions such as automatic media supply, sampling and storage, temperature control, fluorescence and microscopic monitoring, PIV, O2-measurement, etc. To display the functionality we performed a toxicity test with RPTEC cells treated with DMSO in different concentrations.

\section{Proof of concept study}

RPTEC cells were used as cellular model system. The cells were cultivated in two Generation-4-MOCs as well as in 96-well-plates working as reference system. The systems were stained with CellTracker ${ }^{\mathrm{TM}}$ Red and cultivated at $37^{\circ} \mathrm{C}$ and $5 \% \mathrm{CO}_{2}$ saturation. After some hours of resting MOCs and MWPs were treated with $10 \%$ respectively $20 \%$ DMSO. Afterwards the fluorescence activity was measured in 20 minute intervals in order to detect potential cell death. The cells can be detected by the monitoring unit of the robot. A $20 \mu \mathrm{mol} / \mathrm{L}$ CellTracker $^{\mathrm{TM}}$ Red staining provides a sufficient signal which can be monitored over time. The treatment with $10 \%$ DMSO shows a fluorescence signal decline of more than $50 \%$ and the following recovery of them.

\section{Summary}

This project shows the successful development of a robotic platform to handle multi-organ-chips. Maintenance as well as user specific protocols, for example toxicity testing, can be accomplished with a minimum amount of labor time. The MOCs in combination with the robotic platform offer the plug-and-play solution to generate substance interaction data on a Lab-on-Chip system.

\section{Authors' details}

${ }^{1}$ Technische Universität Berlin/Germany. ${ }^{2}$ Fraunhofer IWS, Dresden/Germany. ${ }^{3} \mathrm{GeSiM}$ mbh, Großerkmannsdorf/Germany. ${ }^{4}$ ART Photonics GmbH, Berlin/ Germany.

Published: 4 December 2013

doi:10.1186/1753-6561-7-S6-P28

Cite this article as: Kloke et al: Automated substance testing for lab-onchip devices. BMC Proceedings 2013 7(Suppl 6):P28.

* Correspondence: lutz.kloke@tu-berlin.de

${ }^{1}$ Technische Universität Berlin/Germany

Full list of author information is available at the end of the article

(c) 2013 Kloke et al.; licensee BioMed Central Ltd. This is an Open Access article distributed under the terms of the Creative Commons 